\title{
Measuring subdiffusion parameters
}

\author{
T. Kosztołowicz ${ }^{1}$, K. Dworecki ${ }^{1}$, and St. Mrówczyński ${ }^{1,2}$ \\ ${ }^{1}$ Institute of Physics, Świętokrzyska Academy, ul. Świętokrzyska 15, PL - 25-406 Kielce, Poland \\ ${ }^{2}$ Sottan Institute for Nuclear Studies, ul. Hoża 69, PL - 00-681 Warsaw, Poland
}

(27-th January 2005)

\begin{abstract}
We propose a method to extract from experimental data the subdiffusion parameter $\alpha$ and subdiffusion coefficient $D_{\alpha}$ which are defined by means of the relation $\left\langle x^{2}\right\rangle=\frac{2 D_{\alpha}}{\Gamma(1+\alpha)} t^{\alpha}$ where $\left\langle x^{2}\right\rangle$ denotes a mean square displacement of a random walker starting from $x=0$ at the initial time $t=0$. The method exploits a membrane system where a substance of interest is transported in a solvent from one vessel to another across a thin membrane which plays here only an auxiliary role. Using such a system, we experimentally study a diffusion of glucose and sucrose in a gel solvent. We find a fully analytic solution of the fractional subdiffusion equation with the initial and boundary conditions representing the system under study. Confronting the experimental data with the derived formulas, we show a subdiffusive character of the sugar transport in gel solvent. We precisely determine the parameter $\alpha$, which is smaller than 1 , and the subdiffusion coefficient $D_{\alpha}$.
\end{abstract}

\section{INTRODUCTION}

Subdiffusion occurs in various systems. As examples, we mention here a diffusion in porous media or charge carriers transport in amorphous semiconductors [1-3]. The subdiffusion is characterized by a time dependence of the mean square displacement of a Brownian particle. When the particle starts form $x=0$ at the initial time $t=0$ this dependence in a one-dimension system is of the form

$$
\left\langle x^{2}\right\rangle=\frac{2 D_{\alpha}}{\Gamma(1+\alpha)} t^{\alpha}
$$

where $D_{\alpha}$ is the subdiffusion coefficient measured in the units $\left[\mathrm{m}^{2} / \mathrm{s}^{\alpha}\right]$; the parameter $\alpha$, which we call here as a subdiffusion parameter, obeys $0<\alpha \leq 1$. For $\alpha=1$ one deals with the normal or Gaussian diffusion. The linear growth of $\left\langle x^{2}\right\rangle$ with $t$, which is characteristic for normal diffusion, results from the Central Limit Theorem applied to many independent jumps of a random walker. The anomalous diffusion occurs when the famous theorem fails to describe the system because the distributions of summed random variables are too broad or the variables are correlated to each other. In physical terms, the subdiffusion is related to infinitely long average time that a random walker waits to make a finite jump. Then, its average displacement squared, which is observed in a finite time interval, is dramatically suppressed.

The subdiffusion has been recently extensively studied, see e.g. [1-7]. While the phenomenon is theoretically rather well understood there are very a few experimental investigations. There is no effective method to experimentally measure the parameters $\alpha$ and $D_{\alpha}$. In the pioneering study [7], where the subdiffusion coefficient was determined experimentally for the first time, the interdiffusion of heavy and light water in a porous medium was observed by means of nuclear magnetic resonance. The subdiffusion coefficient $D_{\alpha}$ was determined, using the special case $\alpha=2 / 3$ solution of the fractional derivative diffusion equation. The procedure is neither very accurate nor of general use.

Our aim here is to develop a method to precisely measure the parameters $\alpha$ and $D_{\alpha}$. For practical reasons, which are explained below, we choose for the experimental study a membrane system containing two vessels with a thin membrane in between which separates the initially homogeneous solute of the substance of interest from the pure solvent. A schematic view of the system is presented in Fig. 1. We show that the membrane does not affect, as expected, the values of investigated parameters. Instead of the mean square displacement (1), our method refers to the temporal evolution of the thickness $\delta$ of the so-called near-membrane layer which is defined as a distance from the membrane where the substance concentration drops $\kappa$ times with respect to the membrane surface; $\kappa$ is an arbitrary number. In our previous paper [8], we demonstrated that for the normal diffusion $\delta(t)=A \sqrt{t}$. Here we are going to study the diffusion of glucose and sucrose in a gel solvent to show that

$$
\delta(t)=A t^{\gamma},
$$

with $\gamma<1 / 2$. Our choice of the gel medium to look for a subdiffusion is far not accidental. A gel is built of large and heavy molecules which form a polymer network. Thus, the gel water solvent resembles a porous material filled 
with water. Since a mobility of sugar molecules is highly limited in such a medium the subdiffusion is expected. As we show below, this expectation is indeed fulfilled.

Using an analytic solution of the fractional subdiffusion equation, we theoretically argue, that $\alpha=2 \gamma$ and that the parameter $A$ uniquely depends, as in the case of the normal diffusion, on the number $\kappa$ and the parameters $\alpha$ and $D_{\alpha}$. Therefore, knowing $A, \kappa$ and $\alpha$, one can deduce $D_{\alpha}$ for arbitrary $\alpha$. Since the subdiffusion parameter $\alpha$ is measured with a high accuracy we can distinguish a very slow normal diffusion from a subdiffusion with $\alpha$ close to unity. Our

very first theoretical and experimental results on the subdiffusion parameter $\alpha$ have been published in two short notes $[9]$.

\section{EXPERIMENT}

The membrane system under investigation is the cuvette of two vessels separated by the horizontally located membrane. Initially, we fill the upper (lower) vessel with the solute of transported substance while in the lower (upper) one there is a pure solvent. Then, the substance diffuses from one vessel to another through the membrane. Since the concentration gradient is in the vertical direction only, the diffusion is expected to be one-dimensional (along the axis $x$ ).

The substance concentration is measured by means of the laser interferometric method. The laser light is split into two beams. The first one goes through the membrane system parallelly to the membrane surface while the second (reference one) goes directly to the light detecting system. The interferograms, which appear due to the interference of the two beams, are controlled by the refraction coefficient of the solute which is turn depends on the substance concentration. The analysis of the interferograms allows one to reconstruct the time-dependent concentration profiles of the substance transported in the system and to find the time evolution of the near-membrane layers which are of our main interest here. We note that the measurement does not disturb the system under study. The experimental set-up is described in detail in [10]. Here we only mention that it consists of the cuvette with membrane, the Mach-Zehnder interferometer including the He-Ne laser, TV-CCD camera, and the computerized data acquisition system.

For each measurement, we prepared two gel samples: the pure gel - $1.5 \%$ water solution of agerose and the same gel dripped by the solute of glucose or sucrose. The concentration of both sugars in the gel was fixed to be either $0.1\left[\mathrm{~mol} / \mathrm{dm}^{3}\right]$ or $0.07\left[\mathrm{~mol} / \mathrm{dm}^{3}\right]$. The two vessels of the membrane system were then filled with the samples and the (slow) processes of the sugar transport across the membrane started. We used an artificial membrane of the thickness below $0.1 \mathrm{~mm}$. The membrane was needed for two reasons. It initially separated the homogeneous sugar solute in one vessel from the pure gel in another one. It also precisely fixed the geometry of the whole system.

When the sugar was diffusing across the membrane we were recording the concentration profiles in the vessel which initially contained pure gel. The examples of typical interferograms and extracted concentration profiles are presented in the earlier paper [10]. The thickness of a near-membrane layer $\delta$ was calculated from the measured concentration profiles $C(x, t)$ according to the definition

$$
C(\delta, t)=\kappa C\left(0^{+}, t\right),
$$

where $\kappa$ is an arbitrary number smaller than unity. In our analysis we used $\kappa=0.12,0.08$ and 0.05 . Computing $\delta$ for various $t$, we found the thickness of near-membrane layer as a function of time.

In Fig. 2 we present $\delta$ as a function of time for the glucose and sucrose transported in a gel for the initial sugar concentration equal $0.1\left[\mathrm{~mol} / \mathrm{dm}^{3}\right]$. For the glucose we took three values of $\kappa=0.12,0.08$ and 0.05 while for the sucrose $\kappa=0.08$. The errors of $\delta(t)$ shown in the figure were found in the following way. We performed 6 independent measurements of the concentration profiles $C(x, t)$ at the same initial sugar concentration. The thickness of the near membrane layer $\delta(t)$ was found for each concentration profile. We note here that $\delta(t)$ is insensitive to a sizeable error of absolute normalization of $C(x, t)$ as it cancels due to the definition (3). Having 6 values of $\delta$ for every $t$, we obtained the mean value of $\delta$ and the standard error. The final errors shown in Figs. 2 were obtained by further multiplying the standard errors by the Student-Fisher coefficient taken at a confidence level $95 \%$ to include the effect of low statistics.

As seen in Fig. 2, the experimentally found time dependence of $\delta$ is well described by the power function (2) with the common index $\gamma=0.45$. In Fig. 3 we show the same data as in Fig. 3 but in a log-log scale to better test the power law dependence. The lines representing $\sqrt{t}$ are also shown for comparison. It is evident that the measured index $\gamma$ is smaller than 0.5. There are some deviations of our data from the power law dependence at $t<300 \mathrm{~s}$. However, as explained in Sec. IIIB, our theoretical formulas, in particular the power law behavior, are derived in the long time approximation.

We fitted the experimental data shown in Figs. 2, 3 by the power law formula $A t^{\gamma}$, using a standard $\chi^{2}$ procedure. The values of $\chi^{2}$ per degree of freedom were smaller than unity for every value of $\kappa$. The error of the universal index 
$\gamma=0.45$ was found to be 0.005 . The parameter $A$ depends on $\kappa$; it is also different for glucose than for sucrose. We found

$$
\begin{array}{ll}
A=0.091 \pm 0.004 & \text { for } \quad \kappa=0.05, \\
A=0.081 \pm 0.004 & \text { for } \quad \kappa=0.08, \\
A=0.071 \pm 0.004 & \text { for } \quad \kappa=0.12,
\end{array}
$$

for glucose and

$$
A=0.064 \pm 0.003 \quad \text { for } \quad \kappa=0.08 \text {. }
$$

for sucrose.

The fact that $\gamma$ is the same for glucose and sucrose suggests that the index depends only on the solvent i.e. the medium where the subdiffusion occurs. As will be shown in Sec. IV, the subdiffusion coefficients for glucose and for sucrose differ from each other. Such a situation resembles the case of normal diffusion where $\gamma=1$ is universal but the diffusion coefficient changes from one substance to another.

At the end of this section we note that the results of measurements of the near-membrane layers for the initial sugar concentration equal $0.07\left[\mathrm{~mol} / \mathrm{dm}^{3}\right]$ fully coincide with those presented above which were performed for the initial concentration equal $0.1\left[\mathrm{~mol} / \mathrm{dm}^{3}\right]$. This simply reflects the linearity of the subdiffusion equation which is discussed in the next section.

\section{THEORY}

In this section we discuss a theoretical description of the experimental result presented in Sec. II. In particular, we derive the formula which allows one to obtain the subdiffusion coefficient $D_{\alpha}$ from the experimental data.

\section{A. General formulas}

The subdiffusion is described by the equation $[1,11]$

$$
\frac{\partial C(x, t)}{\partial t}=D_{\alpha} \frac{\partial^{1-\alpha}}{\partial t^{1-\alpha}} \frac{\partial^{2} C(x, t)}{\partial x^{2}},
$$

with the Riemann-Liouville fractional derivative defined as

$$
\frac{\partial^{\alpha} f(t)}{\partial t^{\alpha}}=\frac{1}{\Gamma(n-\alpha)} \frac{\partial^{n}}{\partial t^{n}} \int_{0}^{t} d t^{\prime} \frac{f\left(t^{\prime}\right)}{\left(t-t^{\prime}\right)^{1+\alpha-n}},
$$

where $n$ is the smallest integer larger than $\alpha>0$. It is tempting to write down Eq. (8) in a simpler form as

$$
\frac{\partial^{\alpha} C(x, t)}{\partial t^{\alpha}}=D_{\alpha} \frac{\partial^{2} C(x, t)}{\partial x^{2}} .
$$

In general, however, the fractional derivative does not obey the property

$$
\frac{\partial^{\alpha}}{\partial t^{\alpha}} \frac{\partial^{\beta}}{\partial t^{\beta}}=\frac{\partial^{(\alpha+\beta)}}{\partial t^{(\alpha+\beta)}},
$$

and Eq. (8) is not fully equivalent to Eq. (9) [12]. And because the subdiffusion equation as derived in the framework of Continuous Time Random Walk [11] is of the form (8), it has become customary to use Eq. (8) rather than Eq. (9). We also note here that Eq. (8) with the temporal fractional derivative of order $\alpha<1$ corresponds to a 'long' (infinite on average) waiting time of the random walker. This is just the physical situation which is expected in a gel medium built of large and heavy molecules forming a polymer network where mobility of the walker is strongly limited.

We solve Eq. (8) in the region $x>0$ for the following initial concentration

$$
C(x, 0)=\left\{\begin{array}{cl}
C_{0} & , x<0 \\
0 & , x>0 .
\end{array}\right.
$$


Here, $x=0$ is the position of an infinitely thin membrane. In fact, we solve Eq. (8) for the Green's function $G\left(x, t ; x_{0}\right)$ with the initial condition

$$
G\left(x, t=0 ; x_{0}\right)=\delta\left(x-x_{0}\right),
$$

and then, the concentration profiles are calculated using the integral formula

$$
C(x, t)=\int d x_{0} G\left(x, t ; x_{0}\right) C\left(x_{0}, 0\right) .
$$

The Green's function $G\left(x, t ; x_{0}\right)$ gives the probability density to find a random walker at the position $x$ in time $t$; the walker starts from $x_{0}$ at $t=0$.

To find the concentration profile $C(x, t)$ and then time evolution of near-membrane layer $\delta$, we use the relation

$$
G\left(x, t ; x_{0}\right)=\int_{0}^{t} d t^{\prime} J\left(0^{+}, t^{\prime} ; x_{0}\right) G_{\mathrm{ref}}\left(x, t-t^{\prime} ; 0^{+}\right),
$$

where $x>0$ while $x_{0}<0 ; J\left(x, t ; x_{0}\right)$ is the flux associated with $G\left(x, t ; x_{0}\right)$ which for $x=0$ gives the flow across the membrane; $G_{\text {ref }}\left(x, t ; x_{0}\right)$ is the Green's function for the half-space system with $x>0$ and the fully reflecting wall at $x=0$. The integral formula (13), which we call the wall relation, represents one-half of the membrane system as a half-space system with a reflecting wall replacing the membrane. The substance flux, however, does not vanish at the wall but it equals the actual flux in the membrane system. For the case of normal diffusion the wall relation (13) was used in Ref. [14]. In Appendix A we show that it also holds for subdiffusion.

Using the relation (13) and Eq. (12), the concentration profile can be written as

$$
C(x, t)=\int_{0}^{t} d t^{\prime} W\left(t^{\prime}\right) G_{\mathrm{ref}}\left(x, t-t^{\prime} ; 0^{+}\right),
$$

where $x>0$ and the function $W(t)$, which equals

$$
W(t)=\int_{-\infty}^{0} d x_{0} J\left(0^{+}, t ; x_{0}\right) C\left(x_{0}, 0\right),
$$

contains all information about the initial and boundary conditions. The interval of integration in Eq. (15) is due to the initial condition (10).

Since the subdiffusion equation is of the second order with respect to the space variable $x$, it requires two boundary conditions at the membrane. The first one simply assumes the continuity of the flux $J$ flowing through the membrane i.e.

$$
J\left(0^{-}, t\right)=J\left(0^{+}, t\right),
$$

where $J$ is the subdiffusion flux given by the generalized Fick's law [15]. It gets a simple form after Laplace transformation $L\{f(t)\} \equiv \hat{f}(s)=\int_{0}^{\infty} f(t) e^{-s t}$. Then, it reads [15]

$$
\hat{J}(x, s)=-D_{\alpha} s^{1-\alpha} \frac{d \hat{C}(x, s)}{d x} .
$$

There is no obvious choice of the second boundary condition. Therefore, we assume that the missing condition is given by a linear combination of concentrations and flux at the membrane i.e.

$$
b_{1} C\left(0^{-}, t\right)+b_{2} C\left(0^{+}, t\right)+b_{3} J(0, t)=0 .
$$

Since the current is continuous at the membrane there is no point to distinguish $J\left(0^{+}, t\right)$ from $J\left(0^{-}, t\right)$. We note that two boundary conditions discussed in the literature are of the general form (18). The first condition can be formulated as follows: If during a given time interval, $N$ particles reach the membrane, the fraction $\sigma$ of them will be stopped while $(1-\sigma)$ will go through. This condition leads to the boundary condition of the form $[8,16-18]$

$$
C\left(0^{+}, t\right)=\frac{1-\sigma}{1+\sigma} C\left(0^{-}, t\right) .
$$


The second condition assumes that the flux flowing through the membrane is proportional to the difference of concentration at membrane surfaces $[19,20]$

$$
J(0, t)=\lambda\left(C\left(0^{-}, t\right)-C\left(0^{+}, t\right)\right),
$$

where the parameter $\lambda$ controls the membrane permeability. We call the boundary conditions $(16,19)$ and $(16,20)$ as 'A' and 'B', respectively, and in Sec. III C, we use the indices 'A' and 'B' to denote the solutions of the fractional subdiffusion equation obeying the corresponding boundary conditions.

The initial condition (10) combined with the general boundary condition (18) give the Laplace transform of the function $W(t)$, which is defined by Eq. (15), as

$$
\hat{W}(s)=C_{0} \frac{b_{1} \sqrt{D_{\alpha}}}{b_{1}-b_{2}-b_{3} \sqrt{D_{\alpha}} s^{1-\alpha / 2}} \frac{1}{s^{\alpha / 2}}=C_{0} \frac{b_{1} \sqrt{D_{\alpha}}}{b_{1}-b_{2}} \frac{1}{s^{\alpha / 2}} \sum_{k=0}^{\infty} d^{k} s^{k(1-\alpha / 2)}
$$

where

$$
d \equiv \frac{b_{3}}{b_{1}-b_{2}} \sqrt{D_{\alpha}} .
$$

The derivation of Eq. (21) is discussed in Appendix B. Inverting the Laplace transform, we get

$$
W(t)=C_{0} \frac{b_{1} \sqrt{D_{\alpha}}}{b_{1}-b_{2}} \frac{1}{t^{1-\alpha / 2}} \sum_{k=0}^{\infty} \frac{d^{k}}{\Gamma(\alpha / 2-k(1-\alpha / 2))} \frac{1}{t^{k(1-\alpha / 2)}} .
$$

The Green's function $G_{\text {ref }}$, which enters Eq. (14), can be easily obtained by means of the method of images [1] as

$$
G_{\text {ref }}\left(x, t ; x_{0}\right)=G_{0}\left(x, t ; x_{0}\right)+G_{0}\left(-x, t ; x_{0}\right),
$$

with $G_{0}$ being the Green's function for the homogeneous system given by

$$
G_{0}\left(x, t ; x_{0}\right)=\frac{2 \sqrt{D_{\alpha}}}{\alpha\left|x-x_{0}\right|} H_{1}^{1} \frac{1}{1}\left(\left(\frac{\left|x-x_{0}\right|}{\sqrt{D_{\alpha} t^{\alpha}}}\right)^{\frac{2}{\alpha}} \mid \begin{array}{ll}
1 & 1 \\
1 & \frac{2}{\alpha}
\end{array}\right),
$$

where $H$ denotes the Fox function. In our numerical calculations we use the form of $H$

$$
H_{11}^{10}\left(\frac{a^{\frac{1}{\beta}}}{t} \mid \begin{array}{cc}
1 & 1 \\
\frac{1+\nu}{\beta} & \frac{1}{\beta}
\end{array}\right)=\beta\left(\frac{a^{\frac{1}{\beta}}}{t}\right)^{1+\nu} \sum_{k=0}^{\infty} \frac{1}{\Gamma(-k \beta-\nu) k !}\left(-\frac{a}{t^{\beta}}\right)^{k}
$$

which is derived in [13] by means of the Mellin transform technique [22]. In terms of the Laplace transform, the function (24) simplifies to the form [1]

$$
\hat{G}_{0}\left(x, s ; x_{0}\right)=\frac{1}{2 \sqrt{D_{\alpha}} s^{1-\alpha / 2}} e^{-\left|x-x_{0}\right| \sqrt{\frac{s^{\alpha}}{D_{\alpha}}}},
$$

which was found using the formula [13]

$$
L^{-1}\left(s^{\nu} e^{-a s^{\beta}}\right)=\frac{1}{\beta a^{\frac{1+\nu}{\beta}}} H_{1}^{1} 0\left(\frac{a^{\frac{1}{\beta}}}{t} \mid \begin{array}{cc}
1 & 1 \\
\frac{1+\nu}{\beta} & \frac{1}{\beta}
\end{array}\right),
$$

where $a, \beta>0$.

Having the explicit form of the functions $W$ and $G_{\text {ref }}$, we can write down the concentration profile using Eq. (14). The Laplace transform of $C$ equals

$$
\hat{C}(x, s)=C_{0} \frac{b_{1}}{b_{1}-b_{2}-b_{3} \sqrt{D_{\alpha}} s^{1-\alpha / 2}} \frac{1}{s} e^{-x \sqrt{\frac{s^{\alpha}}{D_{\alpha}}}},
$$

which after inverting the transformation can be written down as

$$
C(x, t)=\int_{0}^{t} d t^{\prime} W\left(t-t^{\prime}\right) \frac{2}{\alpha x} H_{11}^{10}\left(\left(\frac{x}{\sqrt{D_{\alpha} t^{\prime \alpha}}}\right)^{\frac{2}{\alpha}} \mid \begin{array}{ll}
1 & 1 \\
1 & \frac{2}{\alpha}
\end{array}\right)
$$

Eq. (29) is the starting point of our further analysis which provides the function $\delta(t)$. 


\section{B. Long time approximation}

We first consider the long time approximation of the formula (29) which, according to the Tauberian theorem [21], corresponds to the small $s$ limit of the Laplace transform. The physical meaning of this approximation is discussed below. Taking into account only the leading contribution in the small $s$ limit, Eq. (28) gets the form

$$
\hat{C}(x, s)=C_{0} \frac{b_{1}}{b_{1}-b_{2}} \frac{1}{s} e^{-x \sqrt{\frac{s \alpha}{D_{\alpha}}}},
$$

which after inverting Laplace transformation provides

$$
C(x, t)=C_{0} \frac{2 b_{1}}{\left(b_{1}-b_{2}\right) \alpha} H_{11}^{10}\left(\left(\frac{x}{\sqrt{D_{\alpha} t^{\alpha}}}\right)^{\frac{2}{\alpha}} \mid \begin{array}{ll}
1 & 1 \\
0 & \frac{2}{\alpha}
\end{array}\right) .
$$

The solution (31) can be also obtained directly from Eq. (29), taking into account only the $k=0$ term in the expansion of $W(t)$ given by Eq. (22), and using the formula

$$
\frac{\sqrt{D_{\alpha}}}{x \Gamma(\alpha / 2)} \int_{0}^{t} \frac{d t^{\prime}}{\left(t-t^{\prime}\right)^{1-\alpha / 2}} H_{11}^{10}\left(\left(\frac{x}{\sqrt{D_{\alpha} t^{\prime \alpha}}}\right)^{\frac{2}{\alpha}} \mid \begin{array}{ll}
1 & 1 \\
1 & \frac{2}{\alpha}
\end{array}\right)=H_{11}^{10}\left(\left(\frac{x}{\sqrt{D_{\alpha} t^{\alpha}}}\right)^{\frac{2}{\alpha}} \mid \begin{array}{ll}
1 & 1 \\
0 & \frac{2}{\alpha}
\end{array}\right)
$$

which is derived by means of the Laplace transformation.

The series $(22)$ can be approximated by the first term if $d \ll t^{1-\alpha / 2}$ which gives

$$
\left(\sqrt{D_{\alpha}} \frac{b_{3}}{b_{1}-b_{2}}\right)^{\frac{1}{1-\alpha / 2}} \ll t .
$$

When the boundary condition is of the form (19), the condition (33) is trivially satisfied for any $t$ as $b_{3}=0$ in this case. Even more, as discussed in the next section, the solution (31) is exact for the boundary condition (19). When the boundary condition is of the form (20), we have $\lambda=b_{1} / b_{3}=-b_{2} / b_{3}$, and the condition (33) can be written as

$$
\left(\frac{\sqrt{D_{\alpha}}}{2 \lambda}\right)^{\frac{1}{1-\alpha / 2}} \ll t .
$$

We have first estimated the l.h.s. of Eq. (34), assuming that we deal with the normal diffusion $(\alpha=1)$. For the membranes used in our experiments the parameter $\lambda$ is of order $10^{-2}[\mathrm{~mm} / \mathrm{s}]$ while the coefficient of normal diffusion $D$ is roughly $10^{-5}\left[\mathrm{~mm}^{2} / \mathrm{s}\right]$. Thus, the l.h.s. of Eq. (34) is estimated as $2 \mathrm{~s}$. Since $10 \mathrm{~s}$ is the time step of our measurements which extend to $2500 \mathrm{~s}$, the condition (34) is fulfilled. We have also checked the condition (34) a posteriori, using the values of $\alpha$ and $D_{\alpha}$ found in Sec. IV. The l.h.s. of Eq. (34) is again about $2 \mathrm{~s}$.

Let us now discuss the temporal evolution of near-membrane layers in the long time approximation. Substituting the solution (31) into Eq. (3), which defines the near-membrane layer, we get the equation

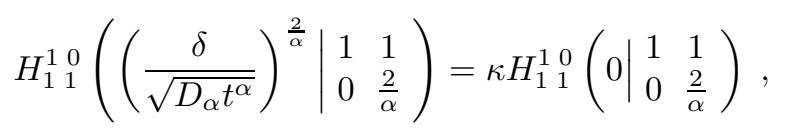

which due to the identity

$$
H_{11}^{10}\left(\begin{array}{l|ll}
0 & 1 & 1 \\
0 & \frac{2}{\alpha}
\end{array}\right)=\frac{\alpha}{2},
$$

simplifies to

$$
H_{11}^{10}\left(\left(\frac{\delta}{\sqrt{D_{\alpha} t^{\alpha}}}\right)^{\frac{2}{\alpha}} \mid \begin{array}{ll}
1 & 1 \\
0 & \frac{2}{\alpha}
\end{array}\right)=\frac{\kappa \alpha}{2} .
$$

One observes that Eq. (36) is solved by

$$
\delta(t)=A\left(\alpha, D_{\alpha}, \kappa\right) t^{\alpha / 2},
$$


where the coefficient $A$ equals

$$
A\left(\alpha, D_{\alpha}, \kappa\right)=\sqrt{D_{\alpha}}\left[\left(H_{1}^{1} \frac{0}{1}\right)^{-1}\left(\frac{\alpha \kappa}{2} \mid \begin{array}{ll}
1 & 1 \\
0 & \frac{2}{\alpha}
\end{array}\right)\right]^{\frac{\alpha}{2}} .
$$

We note that the near-membrane layer given by Eq. (37) does not depend on parameters $b_{1}$ and $b_{2}$ which control membrane permeability. $\delta(t)$ remains the same in the absence of membrane. We also observe that the coefficient $A$ can be recalculated into the diffusion constant $D_{\alpha}$ as

$$
D_{\alpha}=\frac{A^{2}}{\left[\left(H_{1}^{1} \frac{0}{1}\right)^{-1}\left(\frac{\alpha \kappa}{2} \mid \begin{array}{ll}
1 & 1 \\
0 & \frac{2}{\alpha}
\end{array}\right)\right]^{\alpha}} .
$$

Thus, knowing experimental values of $A, \alpha$ and $\kappa$, one can deduce $D_{\alpha}$.

\section{Specific boundary conditions}

In the previous section, we have determined the temporal evolution of near-membrane layer for arbitrary linear boundary condition (18) in the long time approximation. Here we discuss the problem for two specific sets of the boundary conditions $(16,19)$ and $(16,20)$ which are called 'A' and 'B', respectively. The solutions of the subdiffusion equation (8) satisfying the boundary conditions $\mathrm{A}$ and $\mathrm{B}$ can be obtained from the general solution (28), substituting $b_{1}=\sigma+1, \quad b_{2}=\sigma-1, \quad b_{3}=0$ or $b_{1}=\lambda, \quad b_{2}=-\lambda, \quad b_{3}=-1$, respectively. However, the solutions of Eq. (8) with the boundary conditions $\mathrm{A}$ and $\mathrm{B}$ can be also found in a much simpler as demonstrated in Appendix C.

As in the case of normal diffusion, the boundary conditions $(16,19)$ allow for analytic solution of Eq. (8). Using the technique of Laplace transform (see Appendix $\mathrm{C}$ for details), the concentration profile for $x>0$ is found as

$$
C_{\mathrm{A}}(x, t)=(1-\sigma) \frac{C_{0}}{\alpha} H_{1}^{1} \frac{1}{1}\left(\left(\frac{x^{2}}{D_{\alpha} t^{\alpha}}\right)^{\frac{1}{\alpha}} \mid \begin{array}{ll}
1 & 1 \\
0 & \frac{2}{\alpha}
\end{array}\right) .
$$

The solution (40) plugged into the definition of near-membrane layer (3) gives Eq. (36) which is solved by $\delta_{\mathrm{A}}(t)$ of the form (37). Thus, as already mentioned, the formulas derived in the long time approximation are exact for the boundary condition A.

When Eqs. $(16,20)$ are used as the boundary conditions, the solution of subdiffusion equation (8) can be written in the form of the infinite series of Fox functions. As explained in Appendix C, for $x>0$ one finds

$$
C_{\mathrm{B}}(x, t)=\frac{C_{0}}{\alpha} \sum_{n=0}^{\infty}\left[-\frac{x}{2 \lambda}\left(\frac{\sqrt{D_{\alpha}}}{x}\right)^{\frac{2}{\alpha}}\right]^{n} H_{11}^{10}\left(\left(\frac{x^{2}}{D_{\alpha} t^{\alpha}}\right)^{\frac{1}{\alpha}} \mid \begin{array}{cc}
1 & 1 \\
n\left(\frac{2}{\alpha}-1\right) & \frac{2}{\alpha}
\end{array}\right) .
$$

The solutions (40) and (41), which for normal diffusion have been discussed in [16-19], qualitatively differ from each other. In particular, according to Eq. (40) the flux flowing through the membrane is constant in time while the solution (41) leads to the flow which decreases in time as the concentrations at both sides of the membrane evolve to the same value $C_{0} / 2$. In the case of Eq. (40), the ratio of concentrations at both sides is constant in time as dictated by the boundary condition A. However, the qualitative differences of the solutions (40) and (41) are evident for times which are so long that substance concentration becomes approximately homogeneous at each side of the membrane in its neighborhood. These times are usually much longer than those satisfying the condition (33) when the vessels of a membrane system are of a few centimeters length as those used in our measurements.

Since the formula (41) is analytically intractable we have found the time evolution of near-membrane layer numerically. The results are shown in Figs. 4-8. The physical meaning of the near-membrane layer suggests that it does not depend on the membrane permeability. The analytical calculations performed with the boundary condition A fully confirm this intuition. As seen, $\delta_{\mathrm{A}}$ remains the same even in the absence of the membrane $(\sigma=0)$. We are now going to show numerically that the same holds for the boundary condition B. Specifically, we argue that $\delta_{\mathrm{A}}(t)=\delta_{\mathrm{B}}(t)$ for the same values of $\kappa, \alpha$ and $D_{\alpha}$, in spite of the qualitative differences between the solutions (40) and (41).

Fig. 4 demonstrates that $\delta_{\mathrm{B}}(t)$ is independent of the membrane permeability parameter. Our numerical calculations, which are presented in Figs. 5-8, show that the thickness of the near-membrane layer grows in time as $t^{\gamma}$ with $\gamma=\alpha / 2$. Thus, we write

$$
\delta_{\mathrm{B}}(t)=A_{\mathrm{B}} t^{\gamma}
$$


We are now going to show that $A_{\mathrm{A}}=A_{\mathrm{B}}$. In Figs. 5-7, we compare $\delta_{\mathrm{B}}(t)$ obtained numerically with $\delta_{\mathrm{A}}(t)$ given by Eq. (37) for the same values of $\kappa, \alpha$ and $D_{\alpha}$. In each of these figures one parameter changes whereas the remaining two are fixed. In the Fig. 5 we examine the dependence on the parameter defining thickness of near membrane layer $\kappa$; in Fig. 6 there are several values of the subdiffusion coefficient $D_{\alpha}$ while in Fig. 7 the functions are plotted for several values of $\alpha$. In all these cases we see a perfect coincidence of $\delta_{\mathrm{A}}(t)$ with $\delta_{\mathrm{B}}(t)$. Fig. 8 demonstrates that $\delta_{\mathrm{B}}(t)$ fulfills the scaling exactly obeyed by $\delta_{\mathrm{A}}(t)$. Namely, we show that $\delta^{\prime}(t)=\delta_{\mathrm{B}}(t) / A_{\mathrm{A}}$ with $A_{\mathrm{A}}$ given by Eq. (38) depends solely on time, exactly as $\delta_{\mathrm{A}}(t) / A_{\mathrm{A}}$.

We conclude this section by saying that no difference between $\delta_{\mathrm{A}}(t)$ and $\delta_{\mathrm{B}}(t)$ has been observed. Therefore, we expect that $A_{\mathrm{A}}=A_{\mathrm{B}}=A$. Consequently, the subdiffusion coefficients calculated from Eq. (39) have to agree with the ones obtained numerically form the solution (41). Although we are unable to prove beyond the long time approximation that the time evolution of near-membrane layers does not depend on the boundary condition, the results presented in this section strongly substantiate such a conjecture, and thus they justify the use of the formula (39) to evaluate the subdiffusive coefficient $D_{\alpha}$ from experimental data.

\section{EXPERIMENTAL VALUES OF $\alpha$ AND $D_{\alpha}$}

In Sec. II we have fitted the experimentally obtained $\delta(t)$ by the power function $A t^{\gamma}$. Thus, we have found the index $\alpha=2 \gamma=0.90 \pm 0.01$ and the values of the coefficient $A$ given in Eqs. $(4,5,6)$ and $(7)$. Now, we recalculate $A$ into $D_{\alpha}$ by means of the relation (39). Using the numerical values of inverse Fox functions

$$
\begin{gathered}
\left(\begin{array}{ll|l}
H_{1}^{1} & 0
\end{array}\right)^{-1}\left(\begin{array}{ll}
0.054 & \begin{array}{cc}
1 & 1 \\
0 & \frac{1}{0.45}
\end{array}
\end{array}\right)=6.032 \\
\left(\begin{array}{ll}
H_{1}^{1} & 0
\end{array}\right)^{-1}\left(\begin{array}{lll}
0.036 & \begin{array}{cc}
1 & 1 \\
0 & \frac{1}{0.45}
\end{array}
\end{array}\right)=8.014 \\
\left(\begin{array}{ll}
H_{1}^{1} & 0
\end{array}\right)^{-1}\left(\begin{array}{ll}
0.0225 & \begin{array}{ll}
1 & 1 \\
0 & \frac{1}{0.45}
\end{array}
\end{array}\right)=10.510,
\end{gathered}
$$

which are calculated with the help of the expansion formula (25), we computed the subdiffusion coefficient for each value of $\kappa$. As expected, $D_{\alpha}$ shows no dependence on $\kappa$ within the errors which were obtained propagating the errors of $A$. We took the mean value of $D_{\alpha}$ as a final result but the final error is the maximal one. The point is that the values of $D_{\alpha}$ found for different values of $\kappa$ cannot be treated as independent measurements. Thus, we obtained

$$
D_{0.90}=(9.8 \pm 1.0) \times 10^{-4}\left[\mathrm{~mm}^{2} / \mathrm{s}^{0.90}\right]
$$

for glucose and

$$
D_{0.90}=(6.3 \pm 0.9) \times 10^{-4}\left[\mathrm{~mm}^{2} / \mathrm{s}^{0.90}\right]
$$

for sucrose.

To be sure that Eq. (38), which is used to evaluate $D_{\alpha}$, properly describes the experimentally found $\delta(t)$, we checked the scaling property of $\delta(t)$ suggested by the formula (37). In Fig. 9 we plotted the rescaled thickness of near-membrane layer $\delta^{\prime}(t)=\delta(t) / A$ with $A$ given by Eq. (38) for all values of $\kappa$, for glucose and for sucrose. The experimental points are represented as in Fig. 2. According to Eqs. $(37,38), \delta^{\prime}(t)$ is simply the function $t^{\gamma}$, and, as seen in Fig. 9, all our experimental data are indeed very well described by $t^{0.45}$.

\section{FINAL REMARKS}

Our method to determine the parameters of subdiffusion relies on the near-membrane layers. One may ask why $\alpha$ and $D_{\alpha}$ are not extracted directly form the concentration profiles which are measured. There are three reasons to choose the near-membrane layers: experimental, theoretical and practical.

1. Measurement of the near-membrane-layer thickness does not suffer from the sizable $(\sim 10-15 \%)$ systematic uncertainty of absolute normalization of the concentration profiles, as only the relative concentration matters for $\delta$, see the definition (3). 
2. Computed concentration profiles depend on the adopted boundary condition at a membrane while the condition is not well established even for the normal diffusion. The near-membrane layer is argued to be free of this dependence.

3. When the concentration profile is fitted by a solution of the subdiffusion equation, there are three free parameters: $\alpha, D_{\alpha}$ and the parameter characterizing the membrane permeability. Because these fit parameters are correlated with each other it is very difficult in practice to get their unique values. One should remember here that the solution of the subdiffusion equation is of rather complicated structure, see Eqs. (40, 41), which makes the fitting procedure very tedious. When the temporal evolution of $\delta$ is discussed the membrane parameter drops out entirely, $\alpha$ is controlled by the time dependence of $\delta(t)$ while $D_{\alpha}$ is provided by the coefficient $A$.

We have argued using Eq. (8) that $\delta$ scales as $t^{\gamma}$. A similar scaling can be obtained in diffusion equation with fractional derivatives in space and time if the orders of the fractional operators are properly chosen. This is related to the fact that in fractional diffusion equation of order $\mu$ in space and order $\nu$ in time, $\left\langle x^{2}\right\rangle$ scales as $t^{2 \nu / \mu}$. One could also come up with a nonlinear diffusion model without fractional derivatives that exhibits the observed non-Gaussian scaling for $\delta$ [23]. This non-uniqueness problem cannot be solved in full generality but some possibilities can be excluded. The fractional differential equation of the order $\mu<2$ of spatial derivative corresponds to 'long' jumps (the variance is infinite) of the random walker. Since we study experimentally the diffusion in a gel built of large and heavy molecules forming a polymer network, long jumps are physically not plausible. We rather expect that the average waiting time of the random walker in a gel medium goes to infinity. This corresponds to the fractional differential equation with the temporal derivative of order $\alpha<1$ as in Eq. (8). One cannot exclude the equation with fractional spatial and temporal derivatives. Similarly, the nonlinear diffusion equation cannot be excluded. However, Eq. (8), which is derived in a physically well motivated Continuous Time Random Walk approach [1,11], offers the simplest possibility. We also note that the scaling shown in Fig. 9 strongly supports the subdiffusive model based on Eq. (8). It seems difficult to reproduce this scaling in a quite different theory.

In our calculations presented in Sec III, the membrane is assumed to be infinitely thin. Relaxing this assumption considerably complicates theoretical analysis of the problem. This is not only the finite membrane thickness that should be taken into account but the membrane internal structure should be modeled as well. In particular, one should answer the question whether transported substance is accumulated inside the membrane. If so, the membrane permeability might be time dependent. To avoid all these complications, the membrane is infinitely thin in our analysis which is a reasonable assumption as long as the membrane is sufficiently thin. (The thickness of the membrane used in our measurements was below $0.1 \mathrm{~mm}$.) However, we expect that our method to determine the parameters $\alpha$ and $D_{\alpha}$ also works for finite width membranes. First of all, the temporal evolution of near-membrane layer was shown to be fully independent of the boundary condition at a membrane in the long time approximation. Our numerical calculations also suggest that $\delta(t)$ does not depend on the membrane. Finally, we observe that for the near-membranelayer (3) only the relative substance concentration at $x=\delta$ and at the membrane surface $(x=0)$ matters. Therefore, it is expected that the membrane properties do not influence the time evolution of near-membrane layer.

Our method to determine the subdiffusion parameters uses the membrane system. While the membrane plays here only an auxiliary role, it should be stressed that the transport in membrane systems is of interest in several fields of technology [24], where the membranes are used as filters, and biophysics [25], where the membrane transport plays a crucial role in the cell physiology. The diffusion in a membrane system is also interesting by itself as a nontrivial stochastic problem, see e.g. $[16-18,20]$. Thus, our study of the subdiffusion in a membrane system, which to our best knowledge has not been investigated by other authors, opens up a new field of interdisciplinary research. It is also worth mentioning that our interferometric set-up can be used to experimentally study the (anomalous) diffusion not only in the membrane systems. In particular, we plan to perform measurements in a system with no membrane where the sugar is transported directly from the water to gel solvent. However, there are problems to keep fixed geometry of the whole system in the course of long lasting diffusion process.

At the end let us summarize our considerations. We have developed a method to extract the subdiffusion parameters $\alpha$ and $D_{\alpha}$ from experimental data. The method uses the membrane system, where the transported substance diffuses from one vessel to another, and it relies on the fully analytic solution of the fractional subdiffusion equation. We have applied the method to our experimental data on the glucose and sucrose subdiffusion in a gel solvent and we have precisely determined the parameter $\alpha$ and the subdiffusion coefficient $D_{\alpha}$.

\section{ACKNOWLEDGMENTS}

We are grateful to Sławek Wạsik for help in performing the measurements and in the data analysis. 


\section{APPENDIX A: WALL RELATION}

We derive here the integral relation (13) which after the Laplace transformation reads

$$
\hat{G}_{+-}\left(x, s ; x_{0}\right)=\hat{J}_{+-}\left(0^{+}, s ; x_{0}\right) \hat{G}_{\text {ref }}\left(x, s ; 0^{+}\right) .
$$

We have introduced here the indices + and - which correspond to the signs of $x$ and $x_{0}$. Since $x>0$ and $x_{0}<0$, the Green's function is labeled with +- .

Because the current $J\left(x, s ; x_{0}\right)$ is expressed, in accordance with Eq. (17), as

$$
\hat{J}\left(x, s ; x_{0}\right)=-D_{\alpha} s^{1-\alpha} \frac{d \hat{G}\left(x, s ; x_{0}\right)}{d x},
$$

and the general solution of the subdiffusion equation is, as discussed in the Appendix C, given by Eq. (C2), the current equals

$$
\hat{J}_{+-}\left(0^{+}, s ; x_{0}\right)=\sqrt{D_{\alpha}} s^{1-\alpha / 2} B(s)+\frac{1}{2} e^{x_{0} \sqrt{\frac{s^{\alpha}}{D_{\alpha}}}}=\sqrt{D_{\alpha}} s^{1-\alpha / 2} \hat{G}_{+-}\left(0^{+}, s ; x_{0}\right) .
$$

As the Green's function of the system with the reflecting wall at $x=0$ is given by Eqs. $(23,24,26)$, we have

$$
\hat{G}_{\mathrm{ref}}\left(x, s ; 0^{+}\right)=\frac{1}{\sqrt{D_{\alpha}} s^{1-\alpha / 2}} e^{-x \sqrt{\frac{s^{\alpha}}{D_{\alpha}}}},
$$

and due to Eq. (A3) the expression in the r.h.s. of Eq. (A1) equals

$$
\hat{J}_{+-}\left(0^{+}, s ; x_{0}\right) \hat{G}_{\text {ref }}\left(x, s ; 0^{+}\right)=e^{-x \sqrt{\frac{s^{\alpha}}{D_{\alpha}}}} \hat{G}_{+-}\left(0^{+}, s ; x_{0}\right) .
$$

Eq. (C2) allows one to write down the Green's function from the l.h.s. of Eq. (A1) as

$$
\hat{G}_{+-}\left(x, s ; x_{0}\right)=e^{-x \sqrt{\frac{s^{\alpha}}{D_{\alpha}}}} \hat{G}_{+-}\left(0^{+}, s ; x_{0}\right) .
$$

Comparing Eq. (A5) to Eq. (A6), one finally finds the wall relation (A1).

\section{APPENDIX B: FUNCTION $\hat{W}$}

We derive here Eq. (21). Taking the Laplace transform of the boundary condition 18, replacing the concentration profiles by the Green's functions and putting $x_{0}<0$, we get

$$
b_{1} \hat{G}_{--}\left(0^{-}, s ; x_{0}\right)+b_{2} \hat{G}_{+-}\left(0^{-}, s ; x_{0}\right)+b_{3} \hat{J}\left(0, s ; x_{0}\right)=0 .
$$

Using Eq. (C2), one finds

$$
\begin{aligned}
& \hat{G}_{--}\left(x, s ; x_{0}\right)=A(s) e^{x \sqrt{\frac{s \alpha}{D_{\alpha}}}}+\frac{1}{2 \sqrt{D_{\alpha}} s^{1-\frac{\alpha}{2}}} e^{-\left|x-x_{0}\right| \sqrt{\frac{s \alpha}{D_{\alpha}}}}, \\
& \hat{G}_{+-}\left(x, s ; x_{0}\right)=B(s) e^{-x \sqrt{\frac{s \alpha}{D_{\alpha}}}}+\frac{1}{2 \sqrt{D_{\alpha}} s^{1-\frac{\alpha}{2}}} e^{-\left|x-x_{0}\right| \sqrt{\frac{s \alpha}{D_{\alpha}}}} .
\end{aligned}
$$

The Green's functions (B2,B3) provide, via Eq. (17), the currents

$$
\begin{aligned}
& \hat{J}_{--}\left(0^{-}, s ; x_{0}\right)=\frac{1}{2} e^{x_{0} \sqrt{\frac{s \alpha}{D_{\alpha}}}}-\sqrt{D_{\alpha}} A(s), \\
& \hat{J}_{+-}\left(0^{+}, s ; x_{0}\right)=\frac{1}{2} e^{x_{0} \sqrt{\frac{s^{\alpha}}{D_{\alpha}}}}+\sqrt{D_{\alpha}} B(s) .
\end{aligned}
$$

Due to the current continuity equation (16), $A(s)=-B(s)$ and the current, which enters the boundary condition (B1), equals $\hat{J}_{+-}\left(0^{+}, s ; x_{0}\right)$. Substituting the expressions (B2, B3, B5) into Eq. (B1) and using the equality $A(s)=-B(s)$, one finds

$$
B(s)=\frac{b_{1}+b_{2}+b_{3} \sqrt{D_{\alpha}} s^{1-\alpha / 2}}{2 \sqrt{D_{\alpha}} s^{1-\alpha / 2}\left(b_{1}-b_{2}-b_{3} \sqrt{D_{\alpha}} s^{1-\alpha / 2}\right)} e^{x_{0} \sqrt{\frac{s \alpha}{D_{\alpha}}}} .
$$

The function $B(s)$ determines the current (B5) which, substituted into the definition (15) together with the initial condition (10), provides the function $\hat{W}(s)$, as given by Eq. (21). 


\section{APPENDIX C: SOLVING SUBDIFFUSION EQUATION}

We briefly present here a procedure of solving the subdiffusion equation $(8)$ with the boundary conditions $(16,19)$ and, respectively, $(16,20)$. Taking the Laplace transform of Eq. (8), we obtain

$$
\hat{C}(x, s)-s^{-\alpha} D_{\alpha} \frac{d^{2} \hat{C}(x, s)}{d x^{2}}-C(x, 0)=0,
$$

where, as previously, we use the hats to denote the Laplace transformed functions. The solution of Eq. (C1) with the initial condition (11) reads

$$
\hat{G}\left(x, s ; x_{0}\right)=A(s) e^{x \sqrt{\frac{s \alpha}{D_{\alpha}}}}+B(s) e^{-x \sqrt{\frac{s \alpha}{D_{\alpha}}}}+\frac{1}{2 \sqrt{D_{\alpha}} s^{1-\frac{\alpha}{2}}} e^{-\left|x-x_{0}\right| \sqrt{\frac{s \alpha}{D_{\alpha}}}},
$$

where the functions $A(s)$ and $B(s)$ are determined by the boundary conditions.

\section{Boundary conditions A}

The Laplace transform of boundary condition (19) is

$$
\hat{C}\left(0^{+}, s\right)=\frac{1-\sigma}{1+\sigma} \hat{C}\left(0^{-}, s\right) .
$$

Proceeding analogously to the case of normal diffusion [17,18], we find the Green's functions obeying the boundary condition (C3) as

$$
\begin{aligned}
& \hat{G}_{--}\left(x, s ; x_{0}\right)=\hat{G}_{0}\left(x, s ; x_{0}\right)+\sigma \hat{G}_{0}\left(-x, s ; x_{0}\right), \\
& \hat{G}_{+-}\left(x, s ; x_{0}\right)=(1-\sigma) \hat{G}_{0}\left(x, s ; x_{0}\right),
\end{aligned}
$$

where $\hat{G}_{0}$ is the Laplace transform of Green's function for the homogeneous system without a membrane (24); the indices + and - of the Green's functions refer to the sign of $x$ and of $x_{0}$, respectively. form

To compute the concentration profiles we use the Laplace transformed of the integral relation (12) which takes the

$$
\hat{C}(x, s)=\int \hat{G}\left(x, s ; x_{0}\right) C\left(x_{0}, 0\right) d x_{0} .
$$

Now, we substitute the Green's function (C4) into Eq. (C5) and use the formula

$$
L^{-1}\left(s^{\nu} e^{-a s^{\beta}}\right)=\frac{1}{\beta a^{\frac{1+\nu}{\beta}}} H_{11}^{10}\left(\frac{a^{\frac{1}{\beta}}}{t} \mid \begin{array}{cc}
1 & 1 \\
\frac{1+\nu}{\beta} & \frac{1}{\beta}
\end{array}\right),
$$

which is derived in [13] using the Mellin transform technique [22]. Here, $a, \beta>0$ while the parameter $\nu$ is not limited. After simple calculations, we finally find the solution (40).

\section{Boundary conditions B}

The subdiffusion equation (8) is solved by the Green's functions of the form

$$
\begin{aligned}
& \hat{G}_{--}\left(x, s ; x_{0}\right)=A_{1}(s) e^{x \sqrt{\frac{s \alpha}{D_{\alpha}}}}+B_{1}(s) e^{-x \sqrt{\frac{s \alpha}{D_{\alpha}}}}+\frac{1}{2 \sqrt{D_{\alpha}} s^{1-\frac{\alpha}{2}}} e^{-\left|x-x_{0}\right| \sqrt{\frac{s \alpha}{D_{\alpha}}}} \\
& \hat{G}_{+-}\left(x, s ; x_{0}\right)=A_{2}(s) e^{x \sqrt{\frac{s^{\alpha}}{D_{\alpha}}}}+B_{2}(s) e^{-x \sqrt{\frac{s^{\alpha}}{D_{\alpha}}}}+\frac{1}{2 \sqrt{D_{\alpha}} s^{1-\frac{\alpha}{2}}} e^{-\left|x-x_{0}\right| \sqrt{\frac{s^{\alpha}}{D_{\alpha}}}} .
\end{aligned}
$$

The functions $A_{1}(s), A_{2}(s), B_{1}(s)$, and $B_{2}(s)$ are now determined by the boundary conditions $(16,20)$ which after the Laplace transformation take the form 


$$
\begin{aligned}
\hat{J}\left(0^{-}, s ; x_{0}\right) & =\hat{J}\left(0^{+}, s ; x_{0}\right), \\
\hat{J}\left(0, s ; x_{0}\right) & =\lambda\left(\hat{G}_{--}\left(0^{-}, s ; x_{0}\right)-\hat{G}_{+-}\left(0^{+}, s ; x_{0}\right)\right),
\end{aligned}
$$

with the Laplace transform of subdiffusive flux given by the formula [15]

$$
\hat{J}\left(x, s ; x_{0}\right)=-D_{\alpha} s^{1-\alpha} \frac{d \hat{G}\left(x, s ; x_{0}\right)}{d x} .
$$

For the infinite system, one demands vanishing of the Green's functions for $x \rightarrow \pm \infty$, which gives $B_{1} \equiv A_{2} \equiv 0$. Substituting the Green's functions (C7, C8) into the boundary conditions (C9, C10), we obtain

$$
\begin{aligned}
& \hat{G}_{--}\left(x, s ; x_{0}\right)=\frac{1}{4 \lambda+2 \sqrt{D_{\alpha}} s^{1-\frac{\alpha}{2}}} e^{\left(x+x_{0}\right) \sqrt{\frac{s \alpha}{D_{\alpha}}}}+\frac{1}{2 \sqrt{D_{\alpha}} s^{1-\frac{\alpha}{2}}} e^{-\left|x-x_{0}\right| \sqrt{\frac{s \alpha}{D_{\alpha}}}}, \\
& \hat{G}_{+-}\left(x, s ; x_{0}\right)=\frac{4 \lambda}{4 \lambda+2 \sqrt{D_{\alpha}} s^{1-\frac{\alpha}{2}}} \times \frac{1}{2 \sqrt{D_{\alpha}} s^{1-\frac{\alpha}{2}}} e^{-\left(x-x_{0}\right) \sqrt{\frac{s \alpha}{D_{\alpha}}}} .
\end{aligned}
$$

Expanding the function (C11) into the power series with respect to $s$ and using the initial condition (10), the integral relation (C5) provides, with the help of the formula (C6), the solution (41).

[1] R. Metzler and J. Klafter, Phys. Rep. 339, 1 (2000).

[2] R. Metzler and J. Klafter, J. Phys. A 37, R161 (2004); J. Klafter, I.M. Sokolov, and A. Blumen, Phys. Today 55, 48 (2002).

[3] J.P. Bouchaud and A. Georgies, Phys. Rep. 195 (1990), 127.

[4] E. Barkai, R. Metzler, and J. Klafter, Phys. Rev. E 61, 132 (2000).

[5] E. Barkai, Phys. Rev. E 63, 046118 (2001).

[6] S. Lim and S.V. Muniandy, Phys. Rev. E 66, 021114 (2002).

[7] A. Klemm, R. Metzler, and R. Kimmich, Phys. Rev. E 65, 021112 (2002).

[8] K. Dworecki, T. Kosztołowicz, S. Wąsik, and St. Mrówczyński, Eur. J. Phys. E 3, 389 (2000).

[9] K. Dworecki, S. Wassik, and T. Kosztołowicz, Acta Phys. Pol. B 34, 3695 (2003); T. Kosztołowicz and K. Dworecki, Acta Phys. Pol. B 34, 3699 (2003).

[10] K. Dworecki, J. Biol. Phys. 21, 37 (1995).

[11] A. Compte, Phys. Rev. E 53, 4191 (1996).

[12] K.B. Oldham and J. Spanier, The Fractional Calculus (Academic Press, New York, 1974).

[13] T. Kosztołowicz, J. Phys. A 37, 10779 (2004).

[14] O. Sten-Knudsen, Passive Transport Processes, in Membrane Transport in Biology, Vol. I, eds. G. Giebisch, D.C. Tosteson, and H.H. Ussing (Springer, Berlin, 1978), p. 31.

[15] D.H. Zanette, Physica A 252, 159 (1998).

[16] T. Kosztołowicz, Phys. Rev. E 54, 3639 (1996).

[17] T. Kosztołowicz, J. Phys. A 31, 1943 (1998).

[18] T. Kosztołowicz, Physica A 248, 44 (1998).

[19] T. Kosztołowicz and St. Mrówczyński, Acta Phys. Pol. B 32, 217 (2001).

[20] T. Kosztołowicz, Physica A 298, 285 (2001).

[21] I.N. Sneddon, The use of integral transforms, (McGraw, New York, 1972).

[22] W.R. Schneider, in Stochastic Processes in Classical and Quantum Systems, ed. by S. Albeverio, G. Casatti, and D. Merlini (Springer, Berlin, 1986), p. 497.

[23] A. Compte and D. Jou, J. Phys. A 29, 4321 (1996); G. Drazer, H.S. Wio, and C. Tsallis, Granular Matter 3, 105 (2001); E.K. Lenzi, R.S. Mendes, and C. Tsallis, Phys. Rev. E 67, 031104 (2003).

[24] R. Rautenbach and R. Albert, Membrane Processes, (Wiley, Chichester, 1989).

[25] J.H.M. Thornley, Mathematical Models in Plant Physiology, (Academic Press, London, 1976). 


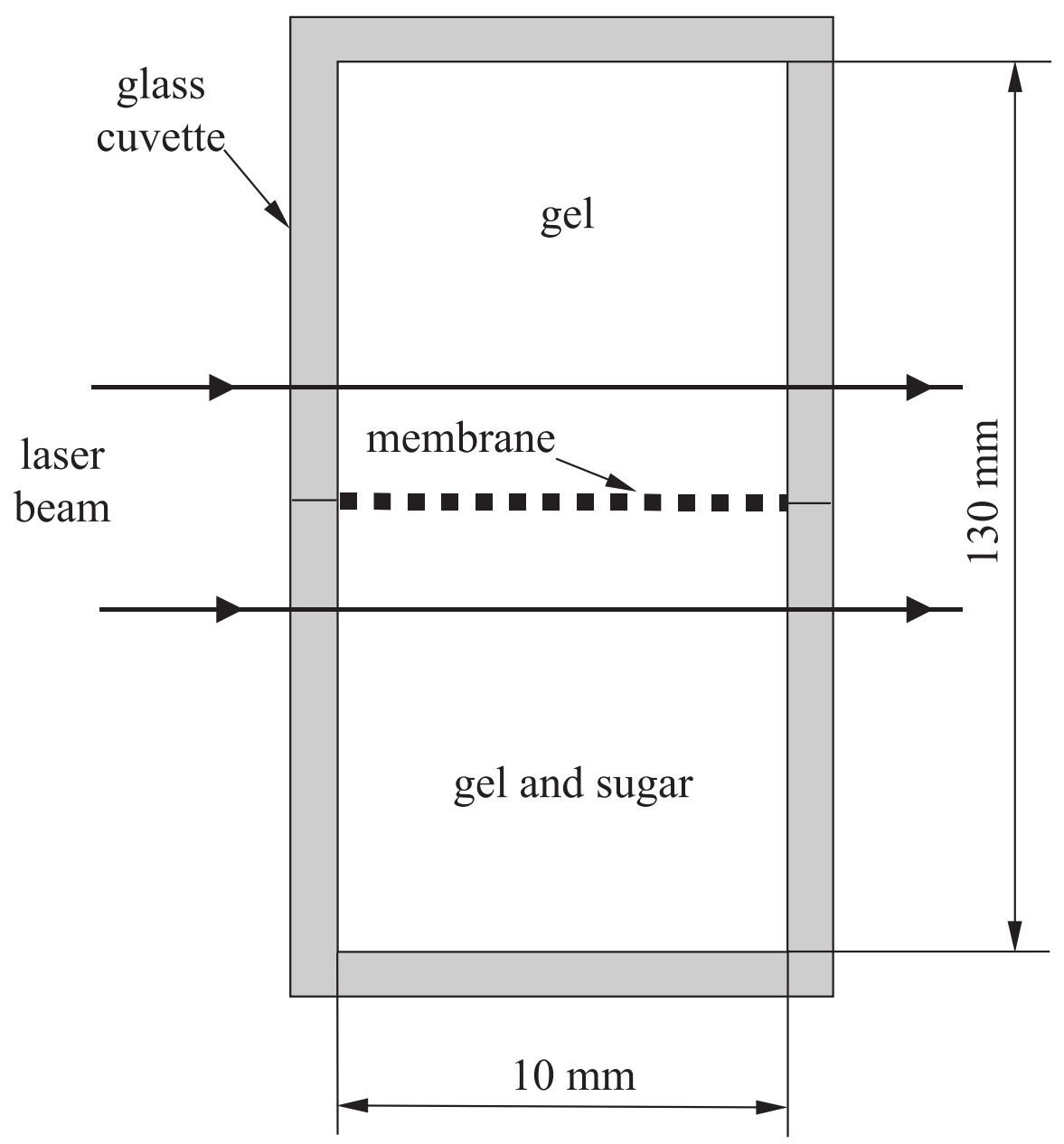

FIG. 1. Schematic view of the membrane system under study. 


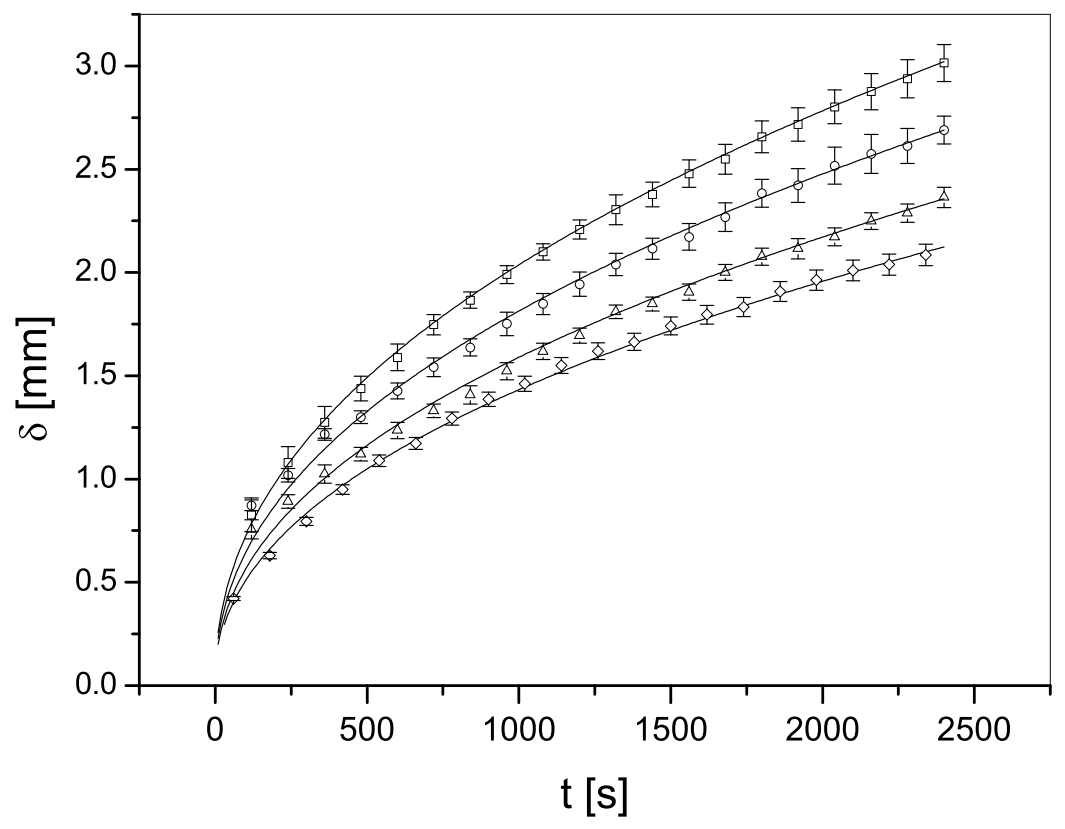

FIG. 2. The experimentally measured thickness of the near-membrane layer $\delta$ as a function of time $t$ for glucose with $\kappa=0.05$ $(\square), \kappa=0.08(\circ), \kappa=0.12(\triangle)$, and for sucrose with $\kappa=0.08(\diamond)$. The lines represent the power function $A t^{0.45}$ with the coefficients $A$ given by Eqs. (4-7).

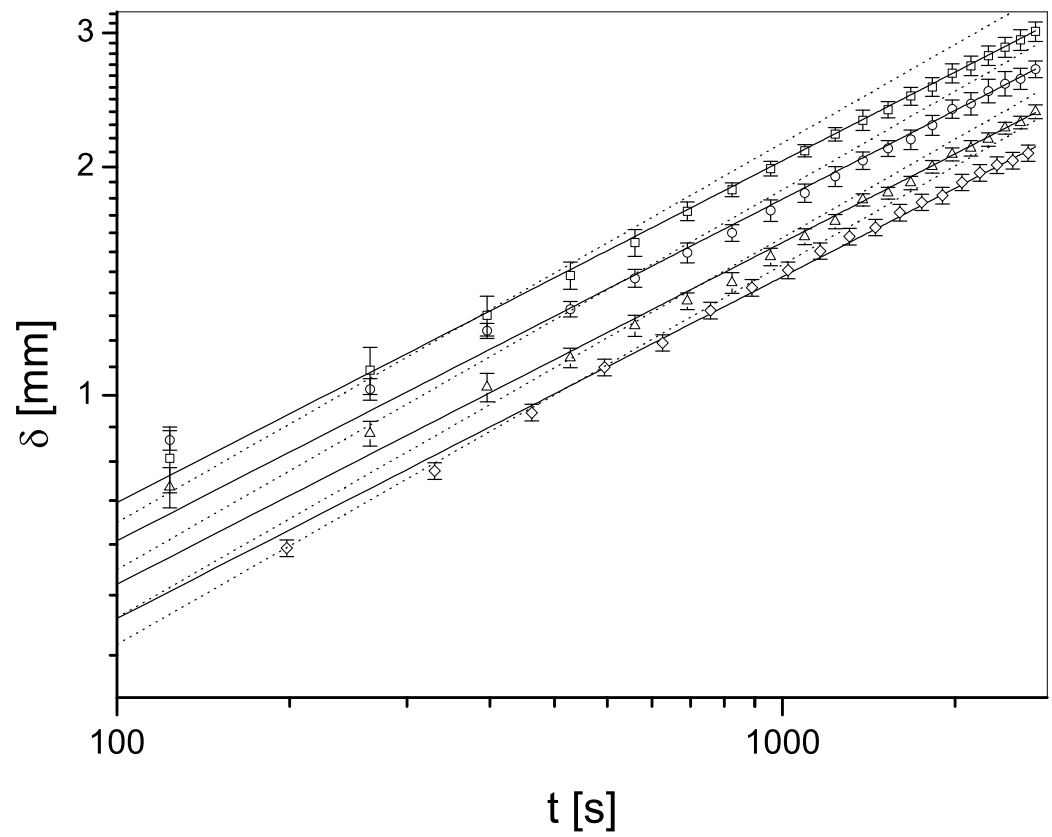

FIG. 3. The same experimental data as in Fig. 2 but in a log-log scale. The solid lines represent the power function $A t^{0.45}$ with the coefficients $A$ given by Eqs. (4-7). The dotted lines correspond to the function $A \sqrt{t}$. 


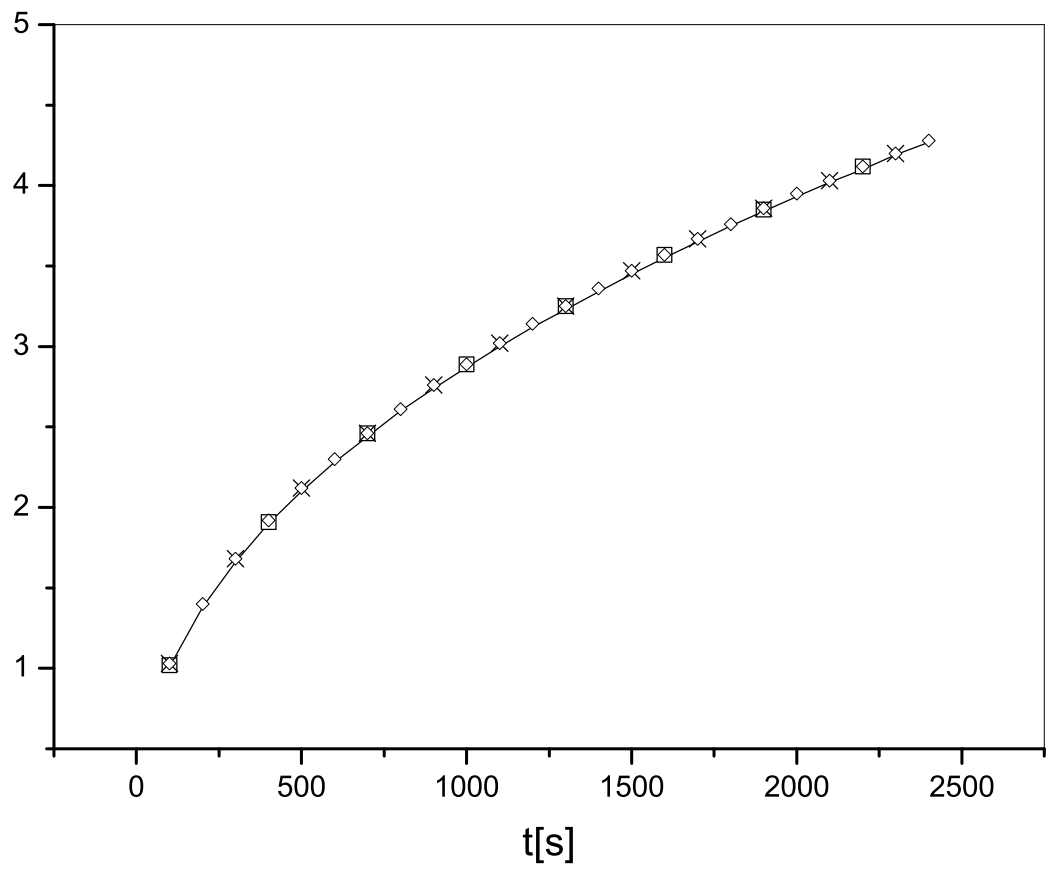

FIG. 4. The time evolution of the near-membrane layer $\delta_{\mathrm{B}}$ obtained from Eq. (41) for different values of the membrane permeability parameter $\lambda: 1[\mathrm{~mm} / \mathrm{s}]$ (solid line), $5[\mathrm{~mm} / \mathrm{s}](\square), 100[\mathrm{~mm} / \mathrm{s}](\times)$, and $500[\mathrm{~mm} / \mathrm{s}](\diamond)$. The remaining parameters equal $\kappa=0.12, \alpha=0.90$, and $D_{0.90}=3 \times 10^{-3} \mathrm{~mm}^{2} / \mathrm{s}^{0.90}$.

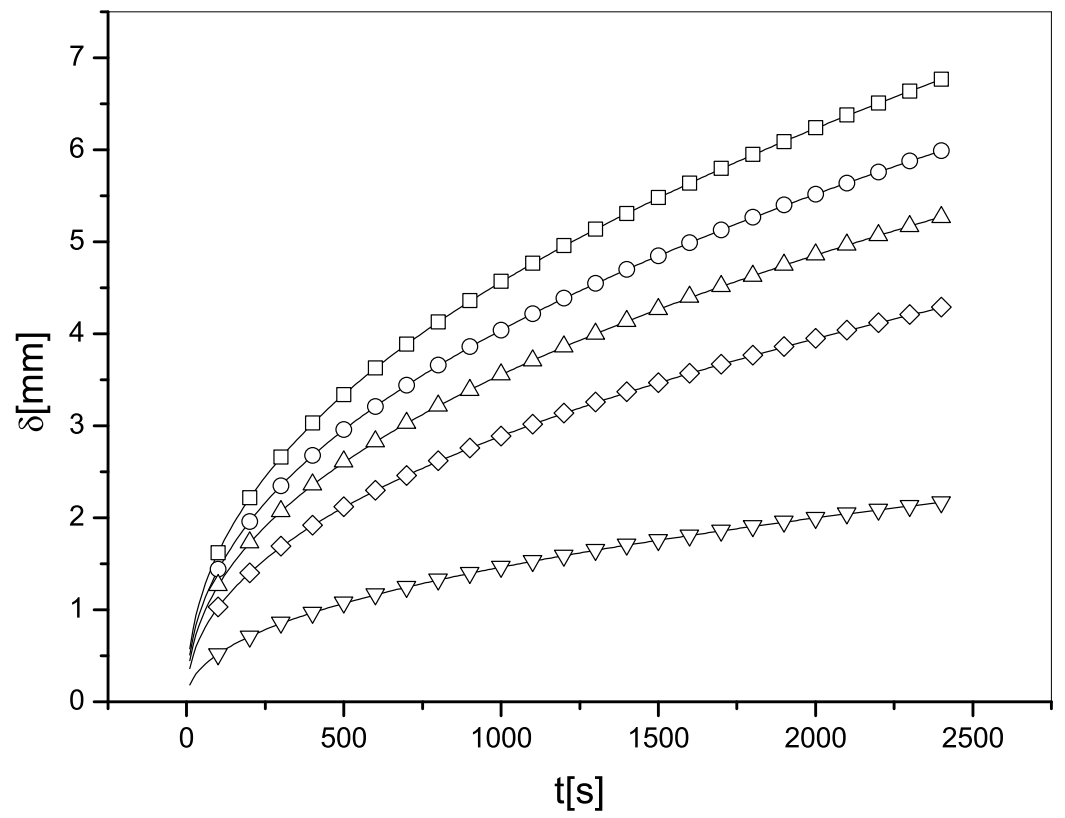

FIG. 5. Comparison of $\delta_{\mathrm{A}}(t)$ (symbols) and $\delta_{\mathrm{B}}(t)$ (lines) for several values of $\kappa: 0.05(\square), 0.08(\circ), 0.12(\triangle), 0.20(\diamond), 0.50$ $(\nabla)$. The remaining parameters equal $\alpha=0.90$ and $D_{0.90}=3 \times 10^{-3} \mathrm{~mm}^{2} / \mathrm{s}^{0.90}$. 


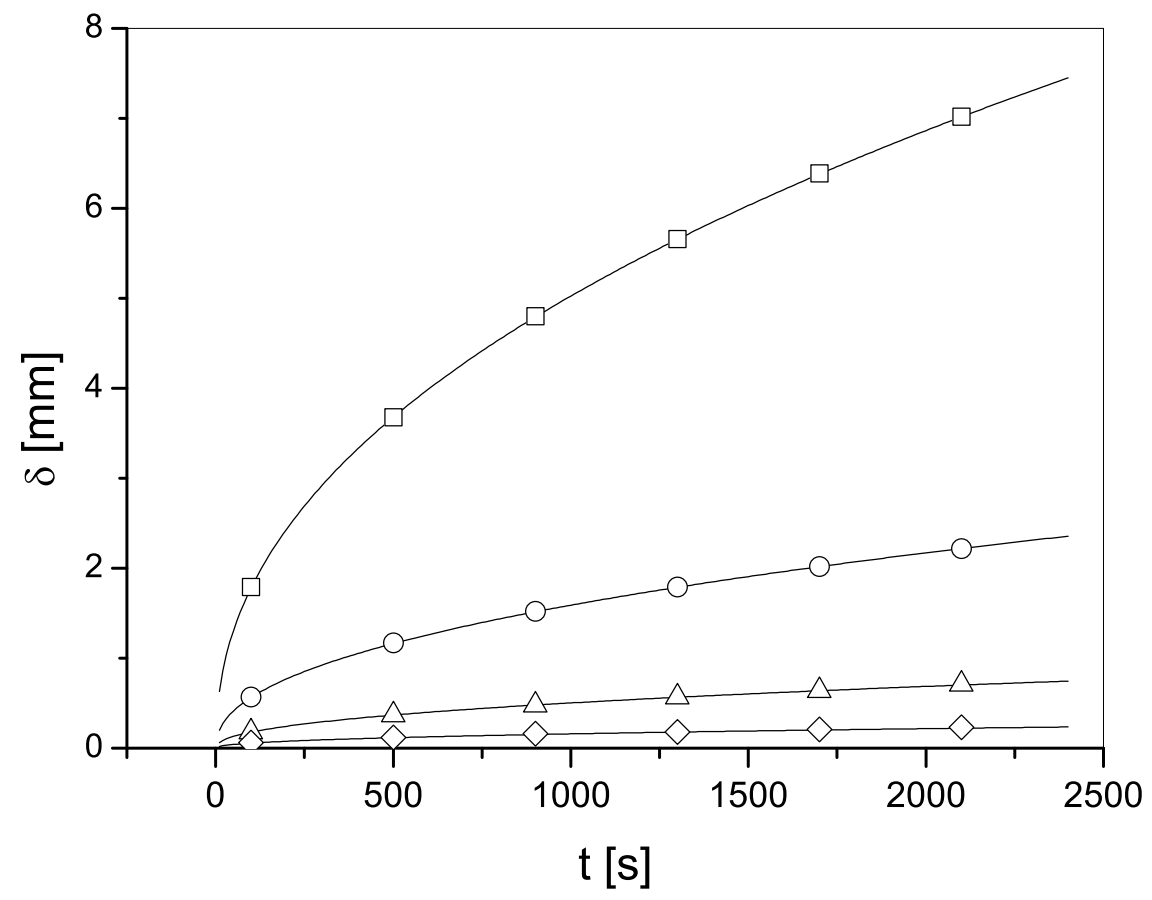

FIG. 6. Comparison of $\delta_{\mathrm{A}}(t)$ (symbols) and $\delta_{\mathrm{B}}(t)$ (lines) for several values of $D_{0.90}: 1 \times 10^{-2} \mathrm{~mm}^{2} / \mathrm{s}^{0.9}(\square), 1 \times 10^{-3} \mathrm{~mm}{ }^{2} / \mathrm{s}^{0.9}$ $(\circ), 1 \times 10^{-4} \mathrm{~mm}^{2} / \mathrm{s}^{0.9}(\triangle), 1 \times 10^{-5} \mathrm{~mm}^{2} / \mathrm{s}^{0.9}(\diamond)$. The remaining parameters equal $\alpha=0.90$ and $\kappa=0.12$.

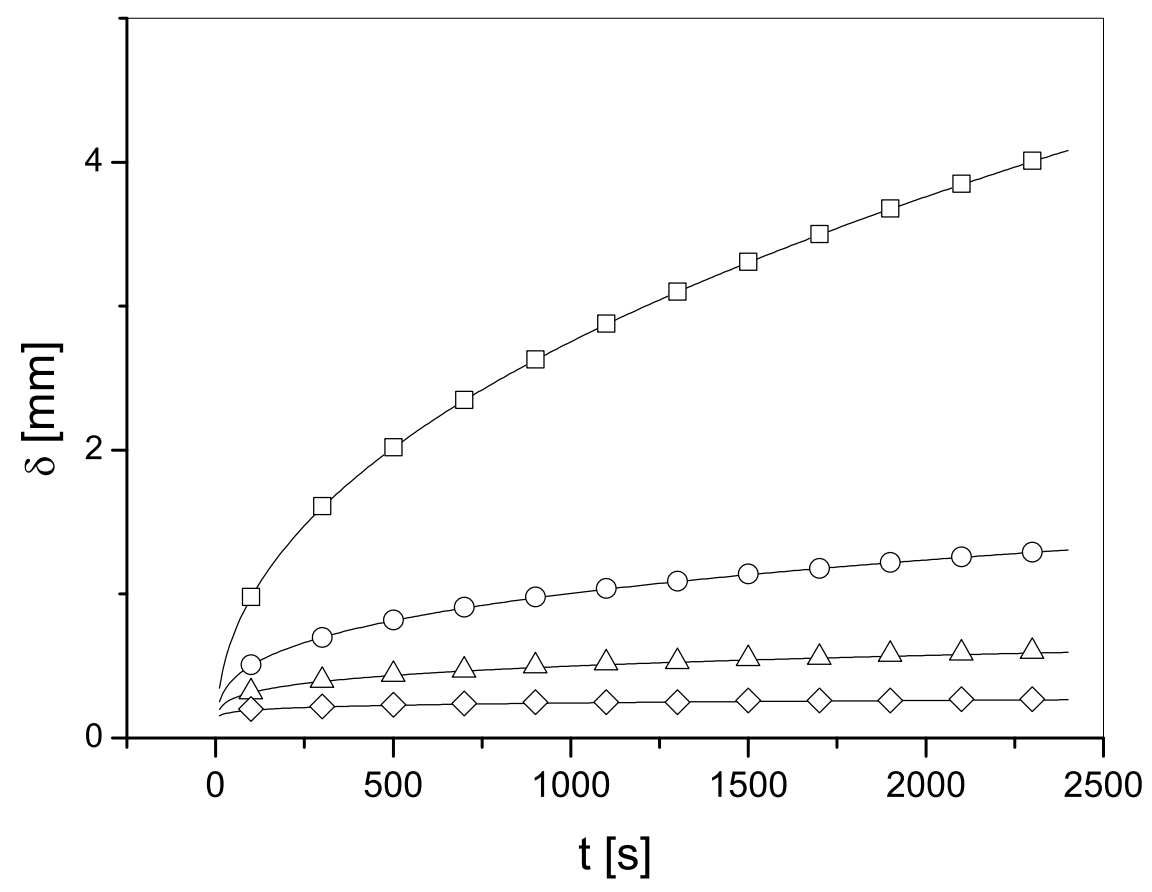

FIG. 7. Comparison of $\delta_{\mathrm{A}}(t)$ (symbols) and $\delta_{\mathrm{B}}(t)$ (lines) for several values of $\alpha$ : $0.9(\square), 0.6(\circ), 0.4(\triangle), 0.2(\diamond)$. The remaining parameters equal $\kappa=0.12$ and $D_{\alpha}=3 \times 10^{-3} \mathrm{~mm}^{2} / \mathrm{s}^{\alpha}$. 


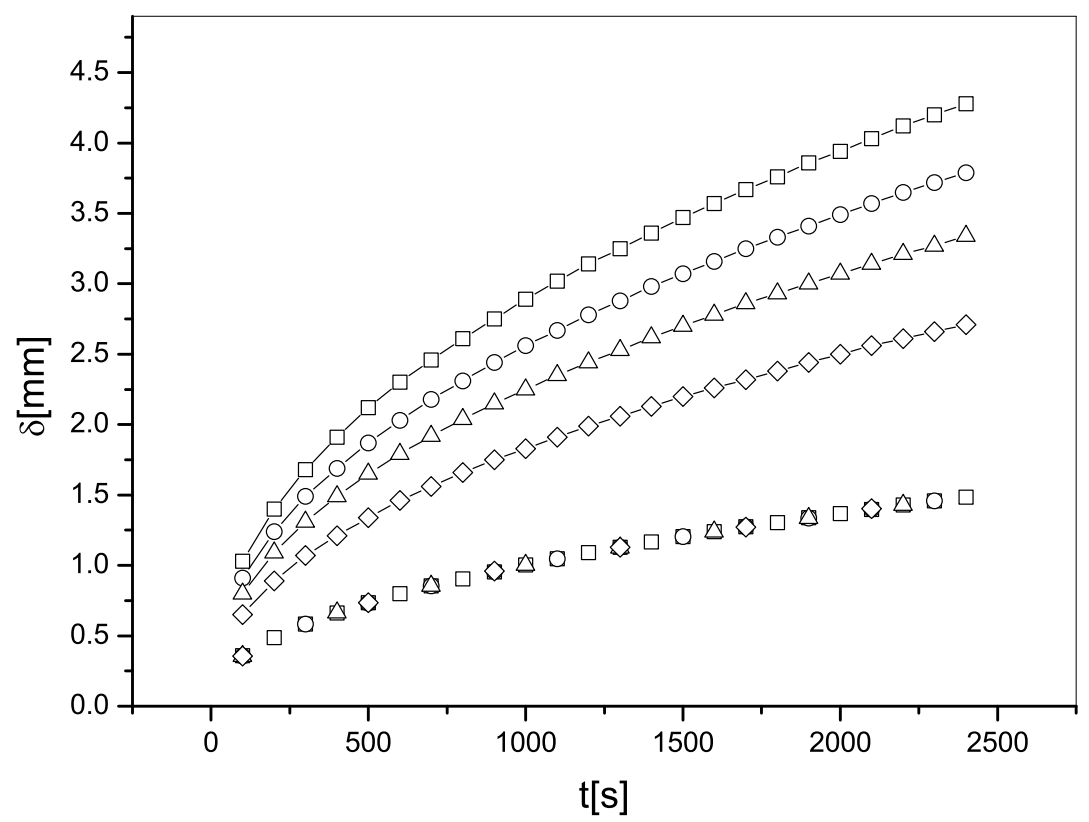

FIG. 8. The thickness of the near-membrane layer $\delta_{\mathrm{B}}$ and the rescaled thickness $\delta_{\mathrm{B}} / A_{\mathrm{A}}$ as functions of time for several values of $\kappa: 0.05(\square), 0.08(\circ), 0.12(\triangle), 0.20(\diamond)$. For the rescaled thickness the symbols are on top of each other. The parameters $\alpha$ and $D_{\alpha}$ equal, respectively, 0.90 and $3 \times 10^{-3} \mathrm{~mm}^{2} / \mathrm{s}^{\alpha}$.

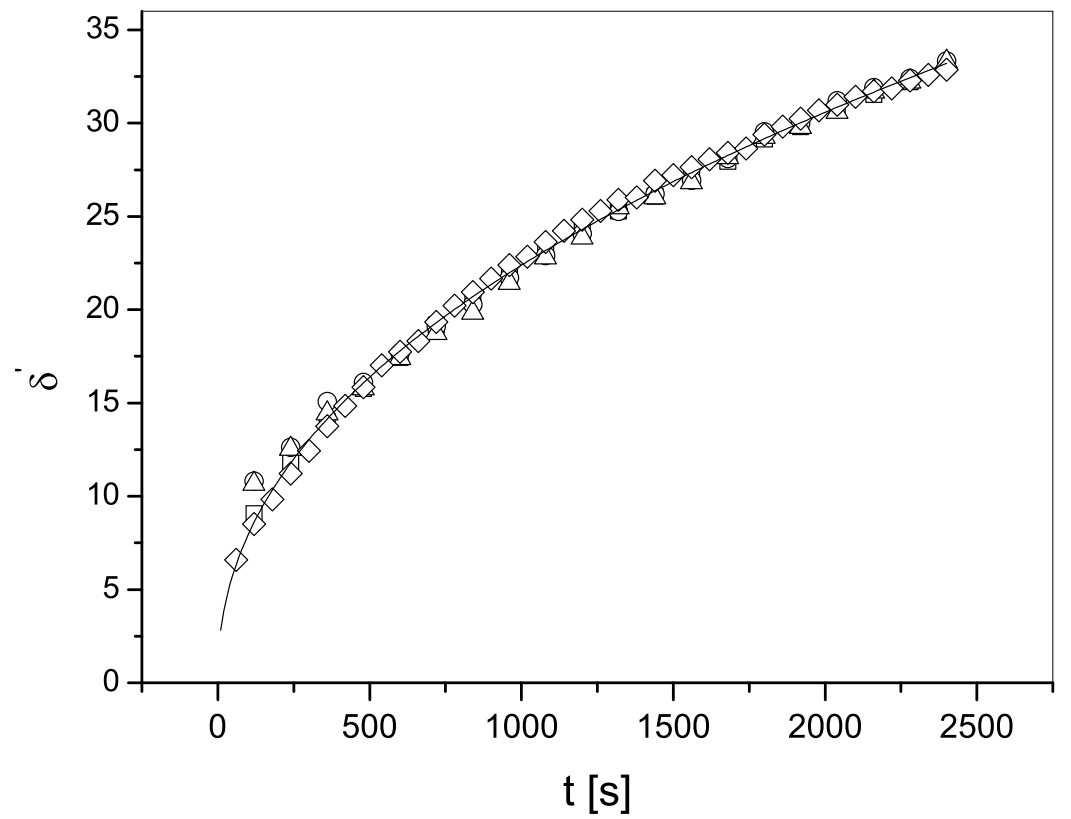

FIG. 9. The experimentally measured $\delta$ divided by the coefficient $A$ given by Eq. (38). The symbols are assigned as in Fig. 2 . The parameters equal $\alpha=0.90$ and $D_{0.90}=1.0 \times 10^{-3}\left[\mathrm{~mm}^{2} / \mathrm{s}^{0.90}\right]$ for glucose and $D_{0.90}=6.3 \times 10^{-3}\left[\mathrm{~mm}^{2} / \mathrm{s}^{0.90}\right]$ for sucrose. For clarity of the plot the error bars are not shown. The line represents the function $t^{0.45}$. 